Artículo

\title{
Bactericera cockerelli vector de Candidatus Liberibacter solanacearum, morfometría y haplotipos en poblaciones de México
}

\author{
Ernesto Cerna Chávez ${ }^{1}$ \\ Mariana Beltrán Beache ${ }^{2}$ \\ Yisa María Ochoa Fuentes ${ }^{3}$ \\ Omegar Hernández Bautista ${ }^{4}$ \\ Juan Carlos Delgado Ortiz ${ }^{5}$
}

${ }^{1}$ Universidad Autónoma Agraria Antonio Narro. Calzada Antonio Narro núm. 1923, Col. Buenavista, Saltillo, Coahuila, México. CP. 25315. (jabaly1@yahoo.com). ${ }^{2}$ Universidad Autónoma de AguascalientesCentro de Ciencias Agropecuarias-Departamento de Fitotecnia. Posta zootécnica, Jesús María, Aguascalientes, México. CP. 20900. (beltranmariana89@gmail.com). ${ }^{3}$ Universidad Autónoma Agraria Antonio Narro. Calzada Antonio Narro núm. 1923. Col. Buenavista, Saltillo, Coahuila, México. (yisa8a@yahoo.com). ${ }^{4}$ Departamento de I+D, CULTA SA de CV. Boulevard Luis Echeverría Álvarez 1700, Col. Altavista, El Mante, Tamaulipas. (omegarhbautista@gmail.com). ${ }^{5}$ CONACYT-Universidad Autónoma Agraria Antonio Narro-Departamento de Parasitología Agrícola. Calzada Antonio Narro núm. 1923, Col. Buenavista, Saltillo, Coahuila, México. CP. 25315. (jdelgado@ conacyt.mx).

${ }^{\S}$ Autor para correspondencia: jdelgado@ conacyt.mx.

\section{Resumen}

Bactericera cockerelli es una plaga de importancia económica en solanáceas en México, por los amarillamientos que causa en los cultivos, así como por la transmisión de Candidatus Liberibacter solanacearum. Se describen variantes genéticas de este insecto, las cuales se relacionan con su capacidad para fungir como vector. En México, la distribución de B. cockerelli es muy amplia y se carece de información acerca de sus características morfológicas y genéticas. El objetivo de esta investigación fue caracterizar morfológica y genéticamente a $B$. cockerelli y detectar la presencia de $C a$. L. solanacearum en poblaciones de $B$. cockerelli de las zonas productoras de solanáceas en México. Para lo cual se muestrearon 35 localidades de 13 estados, sobre cultivos de chile, tomate, berenjena y papa, bajo diferentes sistemas de producción. Se midieron las variables largas de cuerpo (LC), largas de abdomen (LAB) y ancho de abdomen (AAB) en insectos de cada población, se detectó la presencia de $C a$. L. solanacearum en los 13 estados muestreados, donde los machos presentaron el mayor porcentaje de insectos positivos. La presencia de $\mathrm{Ca}$. L. solanacearum no se vio influenciada por el hospedero o el sistema de producción, sino por la presencia de B. cockerelli.

Palabras claves: $C a$. L. solanacearum, berenjena, haplotipo, psílido de la papa.

Recibido: febrero de 2021

Aceptado: mayo de 2021 


\section{Introducción}

Bactericera cockerelli (Hemiptera: Triozidae), conocido también como salerillo, pulgón saltador o psilido de la papa, es una plaga de importancia económica en México, Estados Unidos de América y Nueva Zelanda (Munyaneza, 2010). Su importancia radica en los daños generados, de tipo toxinífero y por la transmisión de procariontes (Ramírez et al., 2012) como Candidatus Liberibacter solanacearum, patógeno de importancia económica en cultivos de solanáceas en México, Honduras, Guatemala, Belice, Estados Unidos de América, Nueva Zelanda, Europa, Noruega, Nicaragua, España, Francia, Suecia, Finlandia y Canadá (Munyaneza, 2013; Hong y Civerolo, 2014; Loiseau et al., 2014; Delgado-Ortiz et al., 2019; Haapalainen et al., 2018; Henrickson et al., 2019).

Las infecciones por $C a$. L. solanacearum han provocado pérdidas millonarias en la industria por la reducción de la producción y calidad de los cultivos (Gudmestad y Secor, 2007). Además de papa, éste patógeno afecta tomate, chile pimiento y otras especies de cultivos de solanáceas (Liefting et $a l ., 2009)$. En tomate se han generado reducciones en la producción hasta en $60 \%$ si se presenta sola o hasta $100 \%$ si se presenta en conjunto con fitoplasmas, mermando la calidad del fruto y su valor comercial (Delgado-Ortiz et al., 2019). Provoca un aumento en los gastos de manejo del cultivo y control del vector (Gudmestad y Secor, 2007; Greenway y Rondon, 2018).

En México los cultivos que han sido afectados por $C a$. L. solanacearum son el cultivo de la papa, chile y tomate en los estados de Sinaloa, Coahuila, Michoacán y en el Estado de México (Camacho-Tapia et al., 2011; Munyaneza et al., 2007, 2009a, 2009b; Rubio et al., 2011). Estudios sobre la variabilidad genética entre poblaciones de B. cockerelli de EUA, han revelado la presencia de dos biotipos que coinciden con la separación geográfica de las poblaciones analizadas, por lo que se denominaron biotipo Occidental (encontrado en Sur de California) y biotipo Central (presente en Colorado y Nebraska) pertenecientes a la misma especie (Liu et al., 2005; Jackson et al., 2009).

La separación de biotipos en insectos se atribuye a la variación entre poblaciones de la misma especie, que pueden incluir características morfológicas, genéticas y físiológicas: como la capacidad de sobrevivir, reproducirse o causar enfermedades en diferentes hospederos (Shufran y Payton, 2009). Un nivel más específico en la clasificación de B. cockerelli, es la de haplotipos; la cual se ha empleado con la finalidad de entender la dinámica poblacional temporal y espacial del psílido mediante análisis genéticos de un solo individuo (Swisher et al., 2012), definiendo un haplotipo como la forma genética que difiere de cualquier otra forma por variaciones en su secuencia de ADN en al menos un nucleótido (Templeton, 2006), donde específicamente, se han descrito cuatro haplotipos de B. cockerelli (Western, Central, Northwestern y Southwestern) en Norte y Centro América (Swisher et al., 2012; 2013; 2014).

La distribución de $B$. cockerelli en México es muy amplia y diversos ejemplares han sido colectados en varias zonas de importancia agrícola de Durango, Tamaulipas, Ciudad de México Michoacán, San Luis Potosí, Morelos, Coahuila, Chihuahua, Nayarit, Jalisco, Baja California, Guanajuato, Aguascalientes, Zacatecas y Sinaloa (Pletsch, 1947; Vega et al., 2008), sin existir registro acerca de sus características morfológicas y genéticas. 
Por lo cual el objetivo de esta investigación fue analizar individuos de B. cockerelli de diversas poblaciones, provenientes de zonas productoras de solanáceas en México, para obtener su caracterización morfológica y genética, así como detectar la presencia de $\mathrm{Ca}$. L. solanacearum.

\section{Materiales y métodos}

Se realizó un recorrido por los estados de Aguascalientes (Ags), Zacatecas (Zac), Jalisco (Jal), Guanajuato (Gto), Hidalgo (Hgo), Puebla (Pue), San Luis Potosí (SLP), Nayarit (Nay), Nuevo León (NL), Durango (Dgo), Michoacán (Mich), Coahuila (Coah) y Tamaulipas (Tamps) de junio a septiembre de 2017, colectado ejemplares de B. cockerelli presentes en cultivos de solanáceas, bajo distintos sistemas de producción (Cuadro 1).

Cuadro 1. Sitios de colecta de B. cockerelli, hospedero y sistema de producción.

\begin{tabular}{|c|c|c|c|c|}
\hline Población & Localidad & Estado & Cultivo & $\begin{array}{l}\text { Sistema de } \\
\text { producción }\end{array}$ \\
\hline 1 & Ojocaliente & $\mathrm{Zac}$ & Tomate & Invernadero \\
\hline 2 & 1/2 Kilo & Ags & Berenjena & Macrotunel \\
\hline 3 & La finca de Adobe, El Taray & Ags & Chile & Cielo abierto \\
\hline 4 & Los Laureles, Villa Hidalgo & Jal & Tomate & Cielo abierto \\
\hline 5 & El Reparito, Villa Hidalgo & Jal & Chile & Invernadero \\
\hline 6 & Potrero de Vaquerías, Calvillo & Ags & Chile & Cielo abierto \\
\hline 7 & Potrero de Vaquerías, Calvillo & Ags & Tomate & Cielo abierto \\
\hline 8 & Potrero de Vaquerías, Calvillo & Ags & Chile & Cielo abierto \\
\hline 9 & Rancho Nuevo de la Luz, León & Gto & Chile & Cielo abierto \\
\hline 10 & San Francisco del Rincón & Gto & Papa & Cielo abierto \\
\hline 11 & Rancho Nuevo de la Luz, León & Gto & Chile, tomate & Cielo abierto \\
\hline 12 & Ciudad Guzmán & Jal & Tomate & Invernadero \\
\hline 13 & San Agustín Metzquititlan & Hgo & Chile & Cielo abierto \\
\hline 14 & San Cristóbal & Hgo & Chile & Cielo abierto \\
\hline 15 & La gallera, Tlaola & Pue & Chile & Cielo abierto \\
\hline 16 & San Juan Tianguismanalco & Pue & Chile & Invernadero \\
\hline 17 & San Juan Tianguismanalco & Pue & Chile & Cielo abierto \\
\hline 18 & La víbora, Villa de Arista & SLP & Chile & Cielo abierto \\
\hline 19 & Bocas & SLP & Tomate & Cielo abierto \\
\hline 20 & Santa Fe, Moctezuma & SLP & Chile & Macrotúnel \\
\hline 21 & Campechana, Villa de Cos & Zac & Chile, tomate & Cielo abierto \\
\hline 22 & Las Catarinas, Fresnillo & $\mathrm{Zac}$ & Papa & Cielo abierto \\
\hline 23 & La Laborcilla, Calera de Víctor Rosales & $\mathrm{Zac}$ & Tomate & Cielo abierto \\
\hline 24 & Santa María del Oro & Nay & Chile, tomate & Invernadero \\
\hline 25 & San Rafael & NL & Papa & Cielo abierto \\
\hline
\end{tabular}




\begin{tabular}{ccccc}
\hline Población & Localidad & Estado & Cultivo & $\begin{array}{c}\text { Sistema de } \\
\text { producción }\end{array}$ \\
\hline 26 & Navidad & NL & Papa & Cielo abierto \\
27 & San Rafael & NL & Tomate & Malla sombra \\
28 & Labor de Abajo, Poanas & Dgo & Chile & Cielo abierto \\
29 & La Borrega, Poanas & Dgo & Chile & Cielo abierto \\
30 & Héctor Márquez, Poanas & Dgo & Tomate & Invernadero \\
31 & El Refugio, Durango & Dgo & Chile & Cielo abierto \\
32 & Cocucho, Charapan & Mich & papa & Cielo abierto \\
33 & Saltillo & Coah & Tomate & Invernadero \\
34 & Hidalgo & Tamps & Chile & Cielo abierto \\
\hline
\end{tabular}

La captura de los insectos se realizó empleando un aspirador manual, mientras que en los sitios donde la presencia de insectos adultos fue limitada, se procedió a colectar follaje infestado con ninfas y huevecillos de B. cockerelli, siendo colocados en tubos de con etanol al $70 \%$.

Para la caracterización morfométrica de $B$. cockerelli se analizaron de forma individual un promedio de 36 adultos colectados de $B$. cockerelli al azar de cada población, para determinar sus dimensiones externas, las variables: la longitud del cuerpo (LC), largo de abdomen (LAB), ancho de abdomen (AAB), largo de ala (LA), ancho de ala (AA) y longitud de antenas (LAN) fueron tomadas con ayuda del programa Dino-Capture 2 en un microscopio estereoscópico SMZ-711 $\left(\right.$ Motic $\left.^{\circledR}\right)$.

La preparación de los insectos adultos conservados en etanol 70\% consistió en colocarlos en una mezcla de glicerol al $90 \%$ para proceder a su montaje sobre portaobjetos exponiendo las partes del insecto a medir. Los datos obtenidos se analizaron por sexo y una comparación de medias entre ambos sexos, con el programa $\mathrm{R}$ Studio versión 3.3, realizando una comparación de medias mediante una prueba de Tukey, $p \leq 0.05$.

Mientras que para la determinación de haplotipos de $B$. cockerelli, a los insectos que previamente se tomaron las medidas corporales e incluyendo ninfas de algunas poblaciones, se extrajo ADN de forma individual empleando la técnica descrita por Doyle y Doyle (1990) modificada. Los haplotipos de $B$. cockerelli se determinaron utilizando los iniciadores CO1 F3 ('5TACGCCATACTAGCAATCGG-3') y CO1 R3 ('5-GAGTAACGTCGTGGTATTCC-3') que amplifican una región de 500 pb del gen mitocondrial Citocromo C Oxidasa subunidad I (Swisher et al., 2012) mediante PCR de punto final, en la reacción de PCR se emplearon $4 \mu 1$ de Taq\&Go ${ }^{T}$ Mastermix (MP Biomedicals), $0.5 \mu \mathrm{l}$ de cada primer a $10 \mu \mathrm{M}$ y $1 \mu \mathrm{l}(50 \mathrm{ng}) \mathrm{de}$ ADN.

El programa de reacción fue una desnaturalización inicial de $98{ }^{\circ} \mathrm{C}$ durante $30 \mathrm{~s}$, seguida por 30 ciclos de $98{ }^{\circ} \mathrm{C}$ por $10 \mathrm{~s}$, alineación a $56^{\circ} \mathrm{C}$ por $20 \mathrm{~s}$ y extensión a $72^{\circ} \mathrm{C}$ por $30 \mathrm{~s}$, seguidos de una extensión final de $72{ }^{\circ} \mathrm{C}$ durante $7 \mathrm{~min}$, en un termociclador Therm 1000 MaxyGene $^{\mathrm{TM}}$, Axygen ${ }^{\circledR}$. Los amplicones generados se secuenciaron en ambos sentidos por Macrogene, EUA, las secuencias obtenidas se alinearon con el programa Bio Edit y se compararon con el programa BLAST ${ }^{\circledR}$ del Centro Nacional de Información Biotecnológica (NCBI), por sus siglas en inglés. 
Para la detección de Candidatus Liberibacter solanacearum en B. cockerelli, se emplearon los iniciadores Lso TX 16/23 F (5'-AATTTTAGCAAGTTCTAAGGG-3') y Lso TX 16/23 R (5'GGTACCTCCCATATCGC-3') que amplifican una región conservada de 383pb entre el 16S y 23S del ADN ribosomal de 'Ca. L. solanacearum' (Ravindran et al., 2011). La reacción de PCR para Lso TX 16/23 F/Lso TX 16/23 R se llevó a cabo con $4 \mu 1$ de Taq\&Go ${ }^{\mathrm{T}}$ Mastermix (MP Biomedicals), $0.5 \mu \mathrm{l}$ de cada iniciador a $10 \mu \mathrm{M}$ y $1 \mu \mathrm{l}$ de $\mathrm{ADN}$ (50 ng), ajustando a un volumen final de reacción de $20 \mu 1$.

La reacción fue realizada bajo las siguientes condiciones del termociclador (Therm 1000 MaxyGeneTM, Axygen ${ }^{\circledR}$ ): desnaturalización inicial de $98{ }^{\circ} \mathrm{C}$ durante $30 \mathrm{~s}$; seguida por 35 ciclos de $98{ }^{\circ} \mathrm{C}$ por $10 \mathrm{~s}, 56{ }^{\circ} \mathrm{C}$ por $20 \mathrm{~s}$ para su alineación y una extensión de $72{ }^{\circ} \mathrm{C}$ por $30 \mathrm{~s}$, seguidos de una extensión final de $72{ }^{\circ} \mathrm{C}$ durante 7 min. Se seleccionaron productos de PCR representativos de cada población de estudio para secuenciar en Macrogene USA, con la finalidad de respaldar los resultados obtenidos.

\section{Resultados y discusión}

Para la caracterización morfométrica de B. cockerelli se analizó un total de 28 poblaciones de las cuales se obtuvieron las dimensiones corporales de las seis variables seleccionadas (LC, LAB, $\mathrm{AAB}, \mathrm{LA}, \mathrm{AA}, \mathrm{LAN}$ ). En el análisis de las variables para las hembras (Cuadro 2), destacan que los mayores registros en las variables $\mathrm{LC}, \mathrm{LAB}, \mathrm{LA}, \mathrm{AA}$ y $\mathrm{AAB}$ fueron determinados en las poblaciones 2, 6 y 8, mientras que para la variable LAN, las mayores dimensiones fueron registradas en las poblaciones 23 y 26. Destacando la población 34 colectada en Hidalgo, Tamaulipas, que registró las menores dimensiones en las variables LC, LAB, AAB y AA, mientras que la población 14, fue la que presentó menor LA, así como la población 20 (Moctezuma, SLP) que presentaron la menor LAN.

Cuadro 2. Morfometría de hembras de B. cockerelli colectados en campo.

\begin{tabular}{ccccccc}
\hline \multirow{2}{*}{ Población $^{*}$} & \multicolumn{7}{c}{ Característica morfológica** } \\
\cline { 2 - 6 } & LC & LAB & AAB & LA & AA & LAN \\
\hline 2 & $2.05 \mathrm{a}$ & $1.048 \mathrm{a}$ & $0.72 \mathrm{~b}$ & $2.649 \mathrm{ab}$ & $1.025 \mathrm{bc}$ & $0.84 \mathrm{bcde}$ \\
3 & $1.969 \mathrm{abcde}$ & $0.977 \mathrm{ab}$ & $0.682 \mathrm{bc}$ & $2.649 \mathrm{ab}$ & $1.002 \mathrm{bc}$ & $0.879 \mathrm{bcd}$ \\
4 & $1.973 \mathrm{abcd}$ & $0.972 \mathrm{ab}$ & $0.663 \mathrm{bc}$ & $2.604 \mathrm{abc}$ & $1.035 \mathrm{bc}$ & $0.862 \mathrm{bcd}$ \\
5 & $1.801 \mathrm{def}$ & $0.861 \mathrm{bcd}$ & $0.585 \mathrm{bcd}$ & $2.606 \mathrm{abc}$ & $1.001 \mathrm{bc}$ & $0.889 \mathrm{bc}$ \\
6 & $2.012 \mathrm{ab}$ & $0.958 \mathrm{ab}$ & $0.701 \mathrm{~b}$ & $2.665 \mathrm{a}$ & $1.058 \mathrm{~b}$ & $0.912 \mathrm{~b}$ \\
7 & $1.956 \mathrm{abcde}$ & $0.942 \mathrm{ab}$ & $0.632 \mathrm{bcd}$ & $2.622 \mathrm{abc}$ & $1.027 \mathrm{bc}$ & $0.855 \mathrm{bcd}$ \\
8 & $1.749 \mathrm{def}$ & $0.894 \mathrm{bcd}$ & $0.943 \mathrm{a}$ & $2.378 \mathrm{bc}$ & $1.321 \mathrm{a}$ & $0.832 \mathrm{bcde}$ \\
9 & $1.951 \mathrm{abcdef}$ & $0.946 \mathrm{ab}$ & $0.654 \mathrm{bc}$ & $2.618 \mathrm{abc}$ & $1.013 \mathrm{bc}$ & $0.802 \mathrm{cde}$ \\
10 & $1.89 \mathrm{abcdef}$ & $0.952 \mathrm{ab}$ & $0.681 \mathrm{bc}$ & $2.428 \mathrm{bc}$ & $0.959 \mathrm{bc}$ & $0.787 \mathrm{cde}$ \\
11 & $1.82 \mathrm{cdef}$ & $0.893 \mathrm{bcd}$ & $0.611 \mathrm{bcd}$ & $2.413 \mathrm{bc}$ & $0.933 \mathrm{bc}$ & $0.778 \mathrm{de}$ \\
12 & $1.834 \mathrm{bcdef}$ & $0.89 \mathrm{bcd}$ & $0.626 \mathrm{bcd}$ & $2.443 \mathrm{bc}$ & $0.928 \mathrm{bc}$ & $0.789 \mathrm{cde}$ \\
13 & $1.711 \mathrm{ef}$ & $0.831 \mathrm{bcd}$ & $0.614 \mathrm{bcd}$ & $2.331 \mathrm{bc}$ & $0.912 \mathrm{bc}$ & $0.766 \mathrm{de}$ \\
14 & $1.817 \mathrm{cdef}$ & $0.935 \mathrm{ab}$ & $0.608 \mathrm{bcd}$ & $2.281 \mathrm{c}$ & $0.879 \mathrm{c}$ & $0.718 \mathrm{de}$ \\
15 & $1.556 \mathrm{ef}$ & $0.552 \mathrm{~d}$ & $0.529 \mathrm{~cd}$ & $2.412 \mathrm{bc}$ & $0.915 \mathrm{bc}$ & $0.717 \mathrm{de}$ \\
\hline
\end{tabular}




\begin{tabular}{ccccccc}
\hline \multirow{2}{*}{ Población $^{*}$} & \multicolumn{7}{c}{ Característica morfológica** } \\
\cline { 2 - 6 } & LC & LAB & AAB & LA & AA & LAN \\
\hline 16 & 1.849 bcdef & $0.917 \mathrm{~b}$ & $0.614 \mathrm{bcd}$ & $2.461 \mathrm{bc}$ & $0.948 \mathrm{bc}$ & $0.806 \mathrm{cde}$ \\
17 & $1.869 \mathrm{abcdef}$ & $0.933 \mathrm{ab}$ & $0.607 \mathrm{bcd}$ & $2.406 \mathrm{bc}$ & $0.929 \mathrm{bc}$ & $0.766 \mathrm{de}$ \\
18 & $1.802 \mathrm{def}$ & $0.879 \mathrm{bcd}$ & $0.634 \mathrm{bcd}$ & $2.383 \mathrm{bc}$ & $0.912 \mathrm{bc}$ & $0.773 \mathrm{de}$ \\
19 & $1.848 \mathrm{bcdef}$ & $0.903 \mathrm{bcd}$ & $0.606 \mathrm{bcd}$ & $2.414 \mathrm{bc}$ & $0.931 \mathrm{bc}$ & $0.726 \mathrm{de}$ \\
20 & $1.808 \mathrm{def}$ & $0.844 \mathrm{bcd}$ & $0.596 \mathrm{bcd}$ & $2.427 \mathrm{bc}$ & $0.95 \mathrm{bc}$ & $0.667 \mathrm{e}$ \\
22 & $1.981 \mathrm{abcd}$ & $0.929 \mathrm{ab}$ & $0.681 \mathrm{bc}$ & $2.637 \mathrm{abc}$ & $1.038 \mathrm{bc}$ & $0.712 \mathrm{de}$ \\
23 & $1.926 \mathrm{abcdef}$ & $0.905 \mathrm{bc}$ & $0.673 \mathrm{bc}$ & $2.573 \mathrm{abc}$ & $1.006 \mathrm{bc}$ & $1.79 \mathrm{a}$ \\
26 & $2.001 \mathrm{abc}$ & $0.963 \mathrm{ab}$ & $0.68 \mathrm{bc}$ & $2.641 \mathrm{abc}$ & $1.019 \mathrm{bc}$ & $1.83 \mathrm{a}$ \\
27 & $1.595 \mathrm{ef}$ & $0.64 \mathrm{~cd}$ & $0.511 \mathrm{~cd}$ & $2.662 \mathrm{ab}$ & $1.002 \mathrm{bc}$ & $0.788 \mathrm{cde}$ \\
28 & $1.802 \mathrm{def}$ & $0.805 \mathrm{bcd}$ & $0.53 \mathrm{~cd}$ & $2.469 \mathrm{abc}$ & $0.897 \mathrm{bc}$ & $0.813 \mathrm{bcde}$ \\
30 & $1.956 \mathrm{abcdef}$ & $0.904 \mathrm{bcd}$ & $0.647 \mathrm{bcd}$ & $2.648 \mathrm{abc}$ & $1.051 \mathrm{bc}$ & $0.85 \mathrm{bcde}$ \\
32 & $1.919 \mathrm{abcdef}$ & $0.876 \mathrm{bcd}$ & $0.624 \mathrm{bcd}$ & $2.608 \mathrm{abc}$ & $1.011 \mathrm{bc}$ & $0.759 \mathrm{de}$ \\
34 & $1.47 \mathrm{f}$ & $0.587 \mathrm{~d}$ & $0.409 \mathrm{~d}$ & $2.308 \mathrm{bc}$ & $0.88 \mathrm{c}$ & $0.7 \mathrm{de}$ \\
35 & $1.9 \mathrm{abcdef}$ & $0.842 \mathrm{bcd}$ & $0.551 \mathrm{~cd}$ & $2.508 \mathrm{abc}$ & $0.962 \mathrm{bc}$ & $0.732 \mathrm{de}$ \\
\hline
\end{tabular}

* = las poblaciones $1,21,24,25,29,31$ y 33 no se incluyeron en el análisis al no contar con adultos para la toma de datos. ${ }^{* *}=$ las medias $(\mathrm{mm})$ con la misma letra dentro de cada variable no muestran diferencia significativa (Tukey, $p \leq$ $0.05)$.

Las variables LC, LAB, AAB y LAN en hembras de B. cockerelli presentaron mayor separación de grupos estadísticamente similares, con variaciones de hasta $0.5 \mathrm{~mm}$ entre poblaciones. Las primeras tres variables se relacionan directamente, dado que LAB influye en el tamaño de LC, mientras que las variables $\mathrm{LAB}$ y AAB se relacionan con la carga de huevecillos en las hembras al momento de su captura; respecto a LAN, esta variable responde a otro estímulo, dado que no se observa comportamiento que corresponda al tamaño de LC, por lo que LAN puede estar influenciado por la disponibilidad de alimento más que por el tamaño del insecto, encontrando LAN de menores tamaños en insectos con LC elevados o viceversa.

Para las variables LA y AA la separación de grupos fue menor, por lo que se determina como una variable de tamaño estable entre las poblaciones de hembras analizadas. Las diferencias entre la morfometría, el desarrollo del ciclo biológico y la fisiología en poblaciones de $B$. cockerelli, además de factores climáticos y de alimentación, también se pueden ser influenciados por la exposición a insecticidas o por el sexo, donde el mayor tamaño de las hembras y ciclos de vida cortos corresponden a poblaciones más susceptibles (Cerna-Chávez et al., 2018; Hardstone et al., 2010).

En el análisis de las poblaciones de los machos (Cuadro 3), la población 34, coincide con el análisis de las hembras al registrar las menores dimensiones en las variables LC, LAB y LAN. Mientras que la variable LC mostró ser una variable más homogénea, siendo las poblaciones 2 y 6 las que registraron las mayores dimensiones en las variables LAB, AAB, LA y AA, resultando las poblaciones 23 y 26 con la mayor LAN en los machos analizados. 
Cuadro 3. Morfometría de machos de B. cockerelli colectados en campo.

\begin{tabular}{|c|c|c|c|c|c|c|}
\hline \multirow{2}{*}{ Población* } & \multicolumn{6}{|c|}{ Variable morfológica** } \\
\hline & $\mathrm{LC}$ & LAB & $\mathrm{AAB}$ & LA & $\mathrm{AA}$ & LAN \\
\hline 2 & $2.001 \mathrm{a}$ & $0.995 \mathrm{a}$ & $0.615 \mathrm{a}$ & $2.608 \mathrm{a}$ & $0.991 \mathrm{ab}$ & $0.912 \mathrm{bc}$ \\
\hline 4 & $1.894 \mathrm{a}$ & $0.871 \mathrm{ab}$ & $0.535 \mathrm{ab}$ & $2.56 \mathrm{ab}$ & $0.996 \mathrm{ab}$ & $0.903 \mathrm{bc}$ \\
\hline 5 & $1.708 \mathrm{ab}$ & $0.779 \mathrm{ab}$ & $0.514 \mathrm{~b}$ & $2.561 \mathrm{ab}$ & $0.978 \mathrm{c}$ & $0.89 \mathrm{bc}$ \\
\hline 6 & $1.968 \mathrm{a}$ & $0.941 \mathrm{ab}$ & $0.667 \mathrm{a}$ & $2.614 \mathrm{a}$ & $1.086 \mathrm{a}$ & $0.852 \mathrm{bc}$ \\
\hline 7 & $2.014 \mathrm{a}$ & $0.973 \mathrm{a}$ & $0.571 \mathrm{ab}$ & $2.557 \mathrm{abc}$ & $0.988 \mathrm{ab}$ & $0.867 \mathrm{bc}$ \\
\hline 8 & $1.92 \mathrm{a}$ & $0.915 \mathrm{ab}$ & $0.565 \mathrm{ab}$ & $2.536 \mathrm{abc}$ & $0.989 \mathrm{ab}$ & $0.81 \mathrm{c}$ \\
\hline 9 & $1.939 \mathrm{a}$ & $0.926 \mathrm{ab}$ & $0.593 \mathrm{a}$ & $2.477 \mathrm{abc}$ & $0.999 \mathrm{ab}$ & $0.864 \mathrm{bc}$ \\
\hline 10 & $1.803 \mathrm{a}$ & $0.868 \mathrm{ab}$ & $0.554 \mathrm{ab}$ & $2.349 \mathrm{c}$ & $0.917 \mathrm{c}$ & $0.803 \mathrm{c}$ \\
\hline 11 & $1.766 \mathrm{a}$ & $0.849 \mathrm{ab}$ & $0.502 \mathrm{~b}$ & $2.252 \mathrm{c}$ & $0.933 \mathrm{c}$ & $0.815 \mathrm{c}$ \\
\hline 12 & $1.639 \mathrm{abc}$ & $0.706 \mathrm{bc}$ & $0.457 \mathrm{bc}$ & $2.314 \mathrm{c}$ & $0.867 \mathrm{c}$ & $0.846 \mathrm{bc}$ \\
\hline 13 & $1.686 \mathrm{ab}$ & $0.783 \mathrm{ab}$ & $0.506 \mathrm{~b}$ & $2.315 \mathrm{c}$ & $0.897 \mathrm{c}$ & $0.807 \mathrm{c}$ \\
\hline 14 & $1.778 \mathrm{a}$ & $0.85 \mathrm{ab}$ & $0.522 b$ & $2.286 \mathrm{c}$ & $0.862 \mathrm{c}$ & $0.744 \mathrm{c}$ \\
\hline 15 & $1.45 \mathrm{bc}$ & $0.573 \mathrm{c}$ & $0.458 \mathrm{bc}$ & $2.293 \mathrm{c}$ & $0.9 \mathrm{c}$ & $0.763 \mathrm{c}$ \\
\hline 16 & $1.821 \mathrm{a}$ & $0.868 \mathrm{ab}$ & $0.492 \mathrm{~b}$ & $2.386 \mathrm{bc}$ & $0.908 \mathrm{c}$ & $1.135 \mathrm{~b}$ \\
\hline 17 & $1.736 \mathrm{ab}$ & $0.797 \mathrm{ab}$ & $0.511 b$ & $2.301 \mathrm{c}$ & $0.8765 \mathrm{c}$ & $0.835 \mathrm{bc}$ \\
\hline 18 & $1.709 \mathrm{ab}$ & $0.812 \mathrm{ab}$ & $0.511 b$ & $2.311 \mathrm{c}$ & $0.881 \mathrm{c}$ & 0.839 bc \\
\hline 19 & $1.783 \mathrm{a}$ & $0.829 \mathrm{ab}$ & $0.456 \mathrm{bc}$ & $2.356 \mathrm{bc}$ & $0.878 \mathrm{c}$ & $0.725 \mathrm{c}$ \\
\hline 20 & $1.769 \mathrm{a}$ & $0.792 \mathrm{ab}$ & $0.462 \mathrm{bc}$ & $2.36 \mathrm{bc}$ & $0.977 \mathrm{c}$ & $0.755 \mathrm{c}$ \\
\hline 22 & $1.94 \mathrm{a}$ & $0.899 \mathrm{ab}$ & $0.523 \mathrm{ab}$ & $2.575 \mathrm{ab}$ & $0.994 \mathrm{ab}$ & $0.741 \mathrm{c}$ \\
\hline 23 & $1.912 \mathrm{a}$ & $0.865 \mathrm{ab}$ & $0.543 \mathrm{ab}$ & $2.546 \mathrm{abc}$ & $0.964 \mathrm{c}$ & $1.755 \mathrm{a}$ \\
\hline 26 & $1.899 \mathrm{a}$ & $0.895 \mathrm{ab}$ & $0.57 \mathrm{ab}$ & $2.513 \mathrm{abc}$ & $0.949 \mathrm{c}$ & $1.731 \mathrm{a}$ \\
\hline 27 & $1.985 \mathrm{a}$ & $0.946 \mathrm{ab}$ & $0.584 \mathrm{ab}$ & $2.559 \mathrm{abc}$ & $1.005 \mathrm{ab}$ & $0.716 \mathrm{c}$ \\
\hline 28 & $2.082 \mathrm{a}$ & $1.096 \mathrm{a}$ & $0.729 \mathrm{a}$ & $2.255 \mathrm{c}$ & $0.856 \mathrm{c}$ & $0.577 \mathrm{c}$ \\
\hline 30 & $1.643 \mathrm{abc}$ & $0.714 \mathrm{abc}$ & $0.539 b c$ & $2.507 \mathrm{abc}$ & $0.968 \mathrm{c}$ & $0.808 \mathrm{c}$ \\
\hline 32 & $1.753 \mathrm{ab}$ & $0.789 \mathrm{ab}$ & $0.451 \mathrm{ab}$ & $2.476 \mathrm{abc}$ & $0.94 \mathrm{c}$ & $0.798 \mathrm{c}$ \\
\hline 34 & $1.436 \mathrm{c}$ & $0.575 \mathrm{c}$ & $0.409 \mathrm{c}$ & $2.277 \mathrm{c}$ & $0.866 \mathrm{c}$ & $0.733 \mathrm{c}$ \\
\hline 35 & $1.787 \mathrm{a}$ & $0.793 \mathrm{ab}$ & 0.469 bc & $2.43 \mathrm{bc}$ & $0.928 \mathrm{c}$ & $0.724 \mathrm{c}$ \\
\hline
\end{tabular}

* = las poblaciones $1,3,21,24,25,29,31$ y 33 no se incluyeron en el análisis al no contar con adultos para la toma de datos. $^{* *}=$ las medias $(\mathrm{mm})$ con la misma letra dentro de cada variable no muestran diferencia significativa (Tukey, $p \leq$ $0.05)$.

En una comparación entre las dimensiones registradas por los insectos de ambos sexos, las hembras registraron mayor tamaño para las variables analizadas Anova (F27, 658; $p \leq 0.05)$ : LC (1.870a, 1.824b); LAB (0.898a, 0.851b); AAB (0.635a, 0.532b); LA (2.509a, 2.441b); AA (0.986a, 0.949b) y LAN (0.888a, 0.895a) para hembras y machos respectivamente. 
En las variables analizadas las hembras presentaron un tamaño superior $( \pm 0.1 \mathrm{~mm})$, siendo el largo de antenas (LAN) la única variable donde no se observó diferencia significativa entre sexos, Vargas et al. (2013) determinaron que la longitud de las antenas se ve afectada en el insecto según el hospedero en el que se desarrolle, mientras que no pudieron asociar la longitud de las antenas al sexo.

La ubicación geográfica y condiciones ambientales no se contemplan como una determinante en el tamaño corporal de las poblaciones de $B$. cockerelli evaluadas, ya que dentro del mismo estado se encuentran poblaciones en dos o tres de los principales grupos mencionados, además de que dichas condiciones se ven alteradas por el sistema de producción bajo el cual se desarrollan.

Sin embargo, la morfometría si es influenciada por el hospedero, donde las mayores proporciones corporales se ubican en insectos alimentados en el cultivo de berenjena, mientras que en papa, chile y tomate, no se observa un comportamiento determinado. Se ha registrado un aumento en la fecundidad, la taza de sobrevivencia, así como un menor tiempo para completar su ciclo de vida cuando se desarrolla $B$. cockerelli en berenjena que en pimiento bajo condiciones de laboratorio (Yang y Liu, 2009).

Los adultos de $B$. cockerelli son pequeños, variando de 1.3-1.9 mm (Liu y Trumble, 2007), pero esta característica puede variar dependiendo de la planta huésped, el origen geográfico de las poblaciones, sexo y si la medición fue realizada con individuos de campo o laboratorio (Butler y Trumble, 2012), Vargas et al. (2013) demostraron que la morfometría de adultos y ninfas de $B$. cockerelli se ve afectada por el cultivar de tomate en que desarrolla.

Los estudios de morfometría son una herramienta que ha demostrado ser muy efectiva para analizar la variación resultante de la fisiología de los individuos (variación del tamaño), propia de la población y probablemente genético (variación de la forma) (Jaramillo et al., 2002), la variación morfométrica se puede utilizar para discriminar 'poblaciones fenotípicas', definidas como grupos con un crecimiento, mortalidad y tasas de reproducción similares (Cadrin, 2000).

En la determinación de haplotipos de B. cockerelli, en los productos de PCR se generaron los amplicones esperados de 500 pb (Figura 1), posterior a la secuenciación, se obtuvo una secuencia de bases idéntica entre las muestras analizadas. Tras la comparación de secuencias en la base de datos del NCBI, se obtuvo un 100\% de similitud con el haplotipo Central (Swisher et al., 2012).

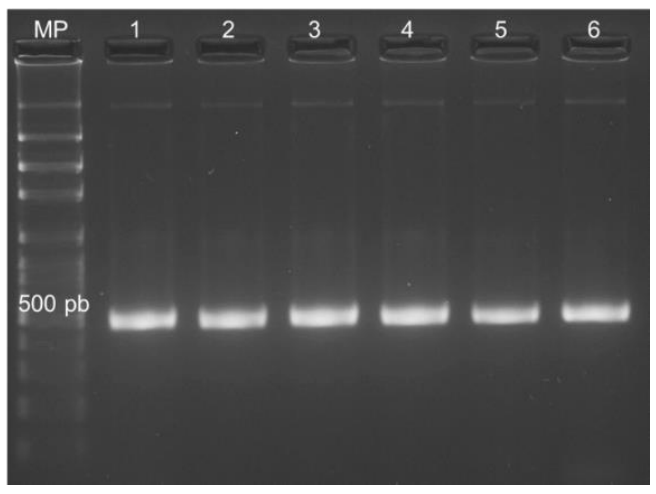

Figura 1. Amplificados obtenidos de B. cockerelli. 
Producto a un peso de 500 pb con los iniciadores CO1 F3/CO1 R3. MP: 100 pb, carril 1: población 8; carril 2: población 18; carril 3: población 24; carril 4: población 28; carril 5: Población 32 y carril 6: población 34; donde cada producto representa a un solo individuo de cada población.

El haplotipo Central se ha localizado en los estados de Wyoming, Nebraska, Texas y Washington en USA; el Salvador, Honduras y Nicaragua en Centro América; en México se ha ubicado en los estados de Toluca y Querétaro (Swisher et al., 2013). El haplotipo Central de B. cockerelli difiere en un solo nucleótido (SNP) con el haplotipo Western en el residuo 51 donde presenta una citosina en sustitución de una timina, así como 16 SNPs con el Northwestern (Swisher et al., 2012) y dos SNPs con el haplotipo Southwestern (Swisher et al., 2014).

En este estudio, se detecta la presencia del haplotipo Central en los estados de Aguascalientes, Jalisco, Durango, Guanajuato, Hidalgo, Puebla, Michoacán, Zacatecas, Nuevo León, Coahuila, Nayarit, San Luis Potosí y Tamaulipas. Liu et al. (2005), reportan dos biotipos de B. cockerelli en el centro y occidente de Estados Unidos de América a los cuales Chapman et al. (2012) separó en Central y Western mediante análisis de fusión de alta resolución y secuenciación de ADN del gen $m t \mathrm{COI}$, estos biotipos presentaron una variación de un nucleótido en la posición 297 de un amplificado de 421 pares de bases, donde el biotipo Central presenta una guanina (G) y el biotipo Western una adenina (A), declarando al biotipo Central el biotipo nativo y al Western como el biotipo invasivo.

De acuerdo con los estudios realizados por Montiel et al. (2016), el biotipo Central (Texas y Nebraska) y el haplotipo Southwestern están estrechamente relacionados, mientras que el biotipo Western, diverge de estas poblaciones, pero se relaciona con una aparente nueva población del noroeste, la cual puede haber surgido a partir de este biotipo.

Para la determinación de $C a$. L. solanacearum en las poblaciones de $B$. cockerelli, se analizaron un total de 862 insectos; 348 hembras, 415 machos y 99 ninfas (obtenidas de 35 poblaciones de 13 estados), de las cuales $80.17 \%$ de las hembras fueron positivas a $\mathrm{Ca}$. L. solanacearum, mientras que en los machos se determinó la presencia de la bacteria en $84.57 \%$ (Cuadro 4).

Cuadro 4. Detección de $C a$. L. solanacearum en poblaciones de B. cockerelli.

\begin{tabular}{ccccc}
\hline \multirow{2}{*}{ Población } & Estado & $\begin{array}{c}\text { Porcentaje de insectos positivos a ' } \mathrm{Ca} . \mathrm{L} . \\
\text { solanacearum' }\end{array}$ & \multicolumn{2}{c}{ Insectos $^{*}$} \\
\hline 1 & Zacatecas & $5 / 5(100)$ & $\mathrm{H}$ & $\mathrm{M}$ \\
2 & Aguascalientes & $33 / 33(100)$ & $18 / 18$ & $15 / 15$ \\
3 & Aguascalientes & $3 / 3(100)$ & $3 / 3$ & \\
4 & Jalisco & $35 / 35(100)$ & $14 / 14$ & $21 / 21$ \\
5 & Jalisco & $0 / 36(0)$ & $0 / 18$ & $0 / 18$ \\
6 & Aguascalientes & $35 / 35(100)$ & $16 / 16$ & $19 / 19$ \\
7 & Aguascalientes & $36 / 36(100)$ & $12 / 12$ & $24 / 24$ \\
8 & Aguascalientes & $32 / 32(100)$ & $10 / 10$ & $22 / 22$ \\
9 & Guanajuato & $36 / 36(100)$ & $11 / 11$ & $25 / 25$ \\
10 & Guanajuato & $35 / 35(100)$ & $12 / 12$ & $23 / 23$ \\
\hline
\end{tabular}




\begin{tabular}{|c|c|c|c|c|}
\hline \multirow{2}{*}{ Población } & \multirow{2}{*}{ Estado } & \multirow{2}{*}{$\begin{array}{l}\text { Porcentaje de insectos positivos a ' } \mathrm{Ca} \text {. L. } \\
\text { solanacearum' }\end{array}$} & \multicolumn{2}{|c|}{ Insectos $^{*}$} \\
\hline & & & $\mathrm{H}$ & $\mathrm{M}$ \\
\hline 11 & Guanajuato & $36 / 36(100)$ & $20 / 20$ & $16 / 16$ \\
\hline 12 & Jalisco & 27/36 (75) & $4 / 9$ & $23 / 27$ \\
\hline 13 & Hidalgo & $16 / 16(100)$ & $10 / 10$ & $6 / 6$ \\
\hline 14 & Hidalgo & $36 / 36(100)$ & $7 / 7$ & $29 / 29$ \\
\hline 15 & Puebla & $13 / 13(100)$ & $1 / 1$ & $12 / 12$ \\
\hline 16 & Puebla & $0 / 14(0)$ & $0 / 6$ & $0 / 8$ \\
\hline 17 & Puebla & $28 / 36(77.7)$ & $20 / 23$ & $8 / 13$ \\
\hline 18 & SLP & $26 / 26(100)$ & $14 / 14$ & $12 / 12$ \\
\hline 19 & SLP & $24 / 24(100)$ & $17 / 17$ & $7 / 7$ \\
\hline 20 & SLP & $17 / 36(47.2)$ & $13 / 22$ & $4 / 14$ \\
\hline 21 & Zacatecas & $23 / 23(100)$ & \multicolumn{2}{|c|}{$\mathrm{N}$} \\
\hline 22 & Zacatecas & $13 / 18(72.2)$ & $6 / 10$ & $7 / 8$ \\
\hline 23 & Zacatecas & $36 / 36(100)$ & $17 / 17$ & $17 / 19$ \\
\hline 24 & Nayarit & $13 / 13(100)$ & \multicolumn{2}{|c|}{$\mathrm{N}$} \\
\hline 25 & Nuevo León & $12 / 12(100)$ & \multicolumn{2}{|c|}{$\mathrm{N}$} \\
\hline 26 & Nuevo León & $23 / 23(100)$ & $15 / 15$ & $8 / 8$ \\
\hline 27 & Nuevo León & $36 / 36(100)$ & $16 / 16$ & $20 / 20$ \\
\hline 28 & Durango & 3/3 (100) & $2 / 2$ & $1 / 1$ \\
\hline 29 & Durango & $3 / 4(75)$ & \multicolumn{2}{|c|}{$\mathrm{N}$} \\
\hline 30 & Durango & $0 / 5(0)$ & $0 / 3$ & $0 / 2$ \\
\hline 31 & Durango & 0/6 (0) & \multicolumn{2}{|c|}{$\mathrm{N}$} \\
\hline 32 & Michoacán & $17 / 17(100)$ & $12 / 12$ & $5 / 5$ \\
\hline 33 & Coahuila & $36 / 36(100)$ & \multicolumn{2}{|c|}{$\mathrm{N}$} \\
\hline 34 & Tamaulipas & $12 / 35(34.2)$ & $4 / 13$ & $8 / 22$ \\
\hline 35 & Puebla & $24 / 36(66.6)$ & $5 / 17$ & $19 / 19$ \\
\hline
\end{tabular}

*H= hembras; $\mathrm{M}=$ machos; $\mathrm{N}=$ ninfas.

Las ninfas de $B$. cockerelli resultaron ser excelentes portadoras de $C a$. L. solanacearum, al encontrarse positivas $100 \%$ las poblaciones donde no fue posible la captura de insectos adultos. En todos los estados muestreados, se obtuvo al menos una población de $B$. cockerelli positiva a $C a$. L. solanacearum, lo cual brinda un amplio panorama acerca de la distribución y relación entre el vector y ' $\mathrm{Ca}$. L. solanacearum'.

En relación con el hospedero sobre el cual se recolectaron las poblaciones de B. cockerelli; $92.3 \%$ de las poblaciones procedentes de tomate, indicaron positivo a $\mathrm{Ca}$. L. solanacearum; mientras que 83.3\% de las muestras procedentes de chile se determinaron positivas a la bacteria. En el caso de las poblaciones colectadas en el cultivo de papa, 100\% de las muestras fueron positivas. 
Siendo que las poblaciones que resultaron negativas a $\mathrm{Ca}$. L. solanacearum bajo sistemas de producción a cielo abierto fueron la población 16, 30 y 31 (Puebla y Durango respectivamente) y la población 5 colectada en invernadero. Thinakaran et al. (2015) han reportado la preferencia del haplotipo Central en campo sobre diferentes hospederos, siendo mayor el favoritismo en cultivos de papa y tomate sobre pimiento, berenjena y Solanaum elaeagnifolium (trompillo), donde las oviposturas se ven favorecidas en el cultivo de papa, mientras que bajo condiciones de laboratorio la preferencia a berenjena sobre el cultivo de tomate y el resto de los hospederos es superior.

Cadena (1993) reportó la presencia de la punta morada de la papa en lotes comerciales de Guanajuato, Nuevo León, Coahuila, Valle de Toluca (Estado de México), Michoacán, Tlaxcala, Hidalgo y Puebla. Coincidiendo con las poblaciones de B. cockerelli positivas a $C a$. L. solanacearum de los estados de Guanajuato, Nuevo León, Coahuila, Michoacán, Hidalgo y Puebla en este estudio, lo cual indica que la enfermedad lleva décadas presente en estas zonas, con la posibilidad de haberse dispersado hacia otras regiones y cultivos, lo cual es respaldado por el resto de las poblaciones de $B$. cockerelli positivas colectadas en los estados de Zacatecas, Aguascalientes, Jalisco, Durango, Nayarit, San Luis Potosí y Tamaulipas.

Investigaciones de Secor y Rivera (2004) documentaron los síntomas de la punta morada en la región papera de Saltillo, Coahuila, cuyo agente etiológico fue determinado por Munyaneza et al. (2007) quien además lo relaciono con la presencia del vector y cuyo reporte incluye al estado de Nuevo León, mientras que Rubio et al. (2011) detectaron a Ca. L. solanacearum en la región papera de Toluca, donde también encontraron la presencia del vector. En el cultivo de tomate, Ca. L. solanacearum ya había sido reportada en los estados de Michoacán (Hernández, 2013) y Sinaloa (Munyaneza et al., 2009a) y en chile pimiento en Sinaloa, México (Munyaneza et al., 2009b).

El grado de tecnificación en la producción de cultivos, generalmente influye en la incidencia de plagas y enfermedades y la severidad del ataque (Jirón-Rojas et al., 2016) y de acuerdo con los resultados de este estudio se puede inferir que tanto el vector como la bacteria son capaces de adaptarse a cualquier sistema de producción al cual tengan acceso. Presentándose ambos, vector y patógeno, en lotes de producción a cielo abierto, mallasombra, macrotúnel e invernadero.

La población proveniente de la localidad de Potrero de Vaquerías, Calvillo, Aguascalientes en cultivo de chile a cielo abierto, que resultó negativa a $\mathrm{Ca}$. L. solanacearum, la cual se encontró contigua a un predio de tomate, cuyo nivel de infestación era considerablemente más elevado que en chile, disminuyendo la posibilidad de que los insectos capturados portaran la bacteria. La población proveniente de El Refugio, Durango, en chile a cielo abierto, en número de insectos obtenidos fue limitada, lo cual disminuyó la probabilidad de tomar aquellos portadores de la bacteria.

El resto de las muestras negativas a $\mathrm{Ca}$. L. solanacearum fueron las provenientes de invernaderos en las localidades de San Juan Tianguismanalco, Puebla y Héctor Márquez, Poanas, Durango, en chile y tomate respectivamente, concluyendo que estas poblaciones no han estado en contacto con individuos u hospederos portadores de la bacteria. 


\section{Conclusiones}

La morfometría presentada de las 28 poblaciones de $B$. cockerelli analizadas representa un gran avance en la caracterización de este importante vector en las principales zonas productoras de solanáceas en México, además de haberse determinado la presencia de $\mathrm{Ca}$. L. solanacearum en las poblaciones de $B$. cockerelli provenientes de trece estados de México. Siendo que todas las poblaciones de $B$. cockerelli se identificaron como haplotipo Central.

\section{Agradecimientos}

Al PhD. Julien Levy de la Universidad de TEXAS A\&M por su valiosa aportación como revisor del presente trabajo. Y al Consejo Nacional de Ciencia y Tecnología (CONACYT) por el apoyo al proyecto de Cátedras-CONACYT 1048.

\section{Literatura citada}

Butler, C. D. and Trumble, J. T. 2012. The potato psyllid, Bactericera cockerelli (Sulc) Hemiptera: Triozidae): life history, relationship to plant diseases, and management strategies. Terrestrial arthropod reviews. 5(1):87-111. https://doi.org/10.1163/187498312X634266.

Cadena, H. M. A. 1993. La punta morada de la papa en México: Incidencia y búsqueda de resistencia. Agrociencia. 4(2):247-256.

Cadrin, S. X. 2000. Advances in morphometric identification of fishery stocks. Reviews in fish Biology and Fisheries. 10(1):91-112. https://doi.org/10.1023/A:1008939104413.

Camacho-Tapia, M.; Rojas-Martínez, R. I.; Zavaleta-Mejía, E.; Hernández-Deheza, M. G.; Carrillo-Salazar, J. A.; Rebollar-Alviter, A. and Ochoa-Martínez, D. L. 2011. Aetiology of chili pepper variegation from Yurécuaro, México. J. Planth Pathol. 93(2):331-335. http://dx.doi.org/10.4454/jpp.v93i2.1187.

Cerna-Chávez, E.; Hernández-Bautista, O.; Ochoa-Fuentes, Y. M.; Landeros-Flores, J.; AguirreUribe, L.A. y Hernández-Juárez, A. 2018. Morfometría de inmaduros y tablas de vida de Bactericera cockerelli (Hemiptera: Triozidae) de poblaciones del noreste de México. Rev. Colomb. Entomol. 44(1):53-60. http://dx.doi.org/10.25100/socolen.v44i1.6543.

Chapman, R. I.; Macias-Velasco, J. F.; Arp, A. P. and Bextine, B. R. 2012. Using quantitative real time PCR melt curve analysis of partial CO1 sequence for rapid biotype differentiation of Bactericera cockerelli (Hemiptera: Triozidae). Southwest. entomology. 37(4):475-484. https://doi.org/10.3958/059.037.0405.

Delgado-Ortíz, J. C.; Beltrán-Beache, M.; Cerna-Chávez, E.; Aguirre-Uribe, L. A.; LanderoFlores, J.; Rodríguez-Pagaza, Y. y Ochoa-Fuentes, Y. 2019. Candidatus liberibacter solanacearum patógeno vascular de solanáceas: Diagnóstico y control. Rev. Especial. Cienc. Químico-Biológicas. 22(1):1-12. https://doi.org/10.22201/fesz.23958723e.2019. 0.177 .

Doyle, J. J. and Doyle, J. L. 1990. Isolation of plant DNA from fresh tissue. Focus. 12(1):13-15.

Greenway, G. A. and Rondon, S. 2018. Economic impacts of zebra chip in idaho, oregon, and washington. American. J. Potato Res. 95(4):362. Doi:10.1007/s12230-018-9636-2.

Gudmestad, N. C. and Secor, G. A. 2007. Zebra chip: a new disease of potato. Nebraska Potato Eyes. 19(1):1-4. 
Hardstone, M. C.; Huang, X.; Harringto, L. C. and Scot, J. G. 2010. Differences in development, glycogen, and lipid content associated with cytochrome P450-mediated permethrin resistance in Culex pipiens quinquefasciatus (Diptera: culicidae). J. Medical Entomol. 47(2):188-198. Doi: 10.1603/me09131.

Henrickson, A.; Kalischuk, M.; Lynn, J.; Meers, S.; Johnson, D. and Kawchuk, L. 2019. First report of zebra chip on potato in Canada. Plant Disease. 103(5):1016-1016. https://doi.org/10.1094/PDIS-09-18-1576-PDN.

Hong, L. and Civerolo, E. L. 2014. Genomics of plant-associated bacteria D. C. Gross, A. lichens-park y C. Kole. Springer. New York. 203-234 p. Doi:10.1007/978-3-64255378-3-9.

Jackson, B. C.; Goolsby, J.; Wyzykowski, A.; Vitovsky, N. and Bextine, B. 2009. Analysis of genetic relationships between potato psyllid (Bactericera cockerelli) populations in the united states, Mexico and guatemala using ITS2 and inter simple sequence repeat (ISSR) data. Plant Sci. 61(1):1-5.

Jirón-Rojas, R. L.; Nava-Camberos, U.; Jiménez-Díaz, F.; Alvarado-Gómez, O. G.; ÁvilaRodríguez, V. y García-Hernández, J. L. 2016. Densidades de Bactericera cockerelli (Sulc) e incidencia del "permanente del tomate" en diferentes condiciones de producción del tomate. Southwestern Entomol. 41(4):1085-1094.

Liefting, L. W.; Sutherland, P. W.; Ward, L. I.; Paice, K. L.; Weir, B. S. and Clover, G. R. 2009. A new 'Candidatus liberibacter'species associated with diseases of solanaceous crops. Plant Disease. 93(3):208-214. https://doi.org/10.1094/PDIS-93-3-0208.

Liu, D. and Trumble, J. T. 2007. Comparative fitness of invasive and native populations of the potato psyllid (Bactericera cockerelli). Entomologia Experimentalis et Applicata. 123(1):35-42. Doi:10.1111/j.1570-7458.2007.00521.x.

Liu, D.; Trumble, J. T. and Stouthamer. R. 2005. Genetic differentiation between eastern populations and recent introductions of potato psyllid (Bactericera cockerelli) into western north America. Entomologia experimentalis et applicata. 118(3):177-183. https://doi.org/10.1111/j.1570-7458.2006.00383.x.

Loiseau, M.; Garnier, S.; Boirin, V.; Merieau, M.; Leguay, A.; Renaudin, I.; Renvoisé, J. P. and Gentit P. 2014. First report of 'Candidatus liberibacter solanacearum' in Carrot in France. Plant Disease. 98(6):839-839. https://doi.org/10.1094/PDIS-08-13-0900-PDN.

Montiel, A. L.; Hail, D.; Macias-Velasco, J. F.; Powell, C. M. and Bextine, B. R. 2016. The mitochondrial genome of the potato psyllid, Bactericera cockerelli sulc., and differences among potato psyllid populations of the United States. Southwestern Entomologist. 41(2):347-360. Doi:10.3958/059.041.0211.

Munyaneza, J. E.; Sengoda, V. G.; Garzón, T. J. A. and Cárdenas, V. O. G. 2009a. First report of "Candidatus liberibacter solanacearum" in tomato plants in Mexico. Plant Disease. 93(10):1076-1076. Doi:10.1094/PDIS-93-10-1076A.

Munyaneza, J. E.; Crosslin, J. M. and Upton, J. E. 2007. Association of bactericera cockerelli (Homoptera: psyllidae) with "zebra chip", a new potato disease in southwestern United States and Mexico. J. Econ. Entomol. 100(3):656-663. Doi: 10.1603/0022-0493(2007) 100[656:aobchp]2.0.co;2.

Munyaneza, J. E. 2010. Psyllids as vectors of emerging bacterial diseases of annual crops. Southwestern entomologist. 35(3):417-477. https://doi.org/10.3958/059.035.0335.

Pletsch, D. J. 1947. The potato psyllid Paratrioza cockerelli (Sulc) its biology and control. Montana Agricultural Experiment Station. Bulletin. 446. 
Ramírez-Davila, J. F.; Porcayo-Camargo, E.; Sánchez-Pale, J. R. and Vázquez-García, L. M. 2012. Spatial behavior comparison of bactericera cockerelli sulc. (Hemiptera: triozidae) in Mexico. Neotropical Entomol. 41(1):9-16. http https://doi.org/10.1007/s13744-012-0019y s://doi.org/10.1007/s13744-012-0019-y.

Ravindran, A.; Levy, J.; Pierson, E. and Gross, D. C. 2011. Development of primers for improved PCR detection of the potato zebra chip pathogen 'Candidatus liberibacter solanacearum'. Plant Disease. 95(12):1542-1546. Doi:10.1094/PDIS-05-11-0386.

Rubio, C.; Almeyda, L.; Cadena, H. and Lobato, S. 2011. Relation between Bactericera cockerelli and presence of candidatus liberibacter psyllaurous in commercial fields of potato. Rev. Mex. Cienc. Agríc. 2(1):17-28.

Secor, G. A. and Rivera-Varas, V. V. 2004. Emerging diseases of cultivated potato and their impact on latin America. Rev. Latinoamericana de la Papa. 1(1):1-8.

Shufran, K. A. and Payton, T. L. 2009. Limited genetic variation within and between Russian wheat aphid (Hemiptera: Aphididae) biotypes in the United States. J. Econ. Entomol. 102(1):440445. Doi:10.1603/029.102.0157.

Swisher, K. D.; Arp, A. P.; Bextine, B. R.; Álvarez, E. A.; Crosslin, J. M. and Munyaneza, J. E. 2013. Haplotyping the potato psyllid, Bactericera cockerelli, in Mexico and central America. Southwestern Entomol. 38(2):201-208. https://doi.org/10.3958/059.038.0205.

Swisher, K. D.; Henne, D. C. and Crosslin, J. M. 2014. Identification of a fourth haplotype of Bactericera cockerelli (Hemiptera: Triozidae) in the United States. Fournal Insect Sci. 14(1):1-7. Doi: 10.1093/jisesa/ieu023.

Swisher, K. D.; Munyaneza, J. E. and Crosslin, J. M. 2012. High resolution melting analysis of the cytochrome oxidase I gene identifies three haplotypes of the potato psyllid in the United States. Environ. Entomol. 41(4):1019-1028. https://doi.org/10.1603/EN12066.

Templeton, A. 2006. Scope and basic premises of population genetics. In: Templeton, A. R. (Ed.). Populations genetics and micro evolutionary theory. John Wiley and Sons, Inc. US. 1-18. p Doi:10.1002/0470047356.ch1.

Thinakaran, J.; Pierson, E. A.; Longnecker, M.; Tamborindeguy, C.; Munyaneza, J. E.; Rush, C. M. and Henne, D. C. 2015. Settling and ovipositional behavior of Bactericera cockerelli (Hemiptera: triozidae) on solanaceous hosts under field and laboratory conditions. J. Econ. Entomol. 108(3):904-916. Doi:10.1093/jee/tov058.

Vargas-Madríz, H.; Bautista-Martínez, N.; Vera-Graziano, J.; García-Gutiérrez, C. and ChavarínPalacio, C. 2013. Morphometrics of eggs, nymphs, and adults of Bactericera cockerelli (Hemiptera: triozidae), grown on two varieties of tomato under greenhouse conditions. Florida Entomologist. 96(1):71-79. https://doi.org/10.1653/024.096.0110.

Vega, G. M. T.; Rodríguez, J. C.; Díaz, G. O.; Bujanos, M. R.; Mota, S. D.; Martínez, C. J. L.; Lagunes, T. A. and Garzón, T. A. 2008. Susceptibilidad a insecticidas en dos poblaciones mexicanas del salerillo, Bactericera cockerelli (Sulc) (Hemiptera: triozidae). Agrociencia. 42(4):463-471.

Yang, X. B. and Liu, T. X. 2009. Life history and life tables of Bactericera cockerelli (Homoptera: Psyllidae) on eggplant and bell pepper. Environ. Entomol. 38(6):1661-1667. Doi:10.1603/022.038.0619. 